Regards sur l'économie allemande

Bulletin économique du CIRAC

$86 \mid 2008$

Varia

\title{
Intérim : un SMIC légal, faute de représentativité syndicale?
}

Isabelle Bourgeois

\section{CpenEdition}

Édition électronique

URL : http://journals.openedition.org/rea/901

DOI : 10.4000/rea.901

ISBN : 978-2-8218-0868-3

ISSN : 1965-0787

\section{Éditeur}

CIRAC

\section{Édition imprimée}

Date de publication : 1 mai 2008

Pagination : 25-36

ISSN : 1156-8992

\section{Référence électronique}

Isabelle Bourgeois, «Intérim : un SMIC légal, faute de représentativité syndicale ? », Regards sur l'économie allemande [En ligne], 86 | mai 2008, document 3, mis en ligne le 01 mai 2010, consulté le 01 mai 2019. URL : http://journals.openedition.org/rea/901 ; DOI : 10.4000/rea.901 


\title{
Intérim : un SMIC légal, faute de représentativité syndicale?
}

\author{
Isabelle Bourgeois
}

L'intérim connaît un véritable boom en Allemagne depuis l'assouplissement de sa réglementation en 2003. Certes, il ne concerne encore guère qu'un peu moins de 800000 personnes, mais sa croissance est particulièrement dynamique. Depuis la légalisation en 1973 de cette forme d'emploi atypique choisie aujourd'hui par quelque $2 \%$ des actifs (un peu plus ou un peu moins selon les sources), le nombre d'intérimaires augmente de $8 \%$ en moyenne annuelle. Sa croissance s'est accélérée durant les années de faiblesse conjoncturelle du début du millénaire, apportant aux entreprises allemandes la flexibilité nécessaire pour répondre aux mutations. Car si, outre-Rhin, l'intérim est aussi (comme en France) un vecteur d'intégration ou de réinsertion professionnelles, il est d'abord et avant tout un pourvoyeur de main-d'œuvre qualifiée apportant just in time les ressources supplémentaires recherchées par les entreprises en période de pic d'activité. En leur garantissant un haut niveau de réactivité, l'intérim est devenu l'un des pivots de la compétitivité de l'économie allemande, car il permet aussi de contrebalancer la rigidité du droit allemand des licenciements.

Or si le travail temporaire a contribué à la flexibilisation de l'emploi décidée dans le cadre de l'Agenda 2010, il fait aujourd'hui l'objet d'un très vif débat dont l'enjeu est le projet de création d'un salaire minimum. Le mouvement syndical allemand met en avant l'argument d'une " précarisation " de l'emploi pour prôner une régulation par l'Etat afin de remédier en réalité à son incapacité à organiser l'intérim selon le modèle traditionnel de la branche et, par-delà, à pallier une représentativité mise à mal par les mutations de l'activité comme la concurrence croissante entre les organisations. Le SPD s'empare de ce dossier pour accroître son audience à l'approche des élections au Bundestag (automne 2009). Et à un an des élections au Parlement européen (juin 2009), des enjeux communautaires alimentent le débat en arrière-plan : la Directive services doit être transcrite en droit national d'ici la fin 2009 et la limitation de la mobilité professionnelle des ressortissants des nouveaux Etats membres entrés en 2004 prend fin en 2011. Enfin, la valeur accordée à la "flexicurité " au sein d'une Stratégie de Lisbonne dont le "volet social " a été augmenté confère une importance nouvelle à un projet de Directive sur le travail intérimaire, sujet d'âpres négociations depuis 1982... Or l'idée d'un SMIC légal, tel qu'il s'applique au BTP depuis dix ans et au secteur postal depuis le $1^{\text {er }}$ janvier de cette année (voir REA 85/08), pourrait s'étendre à toutes les branches : un tel projet de loi est en préparation. S'il est adopté, il scellera définitivement la fin du modèle social contractuel qui est au cœur du "capitalisme rhénan ».

\section{Une réserve flexible de main-d'œuvre qualifiée}

La création d'une entreprise d'intérim étant soumise à autorisation (renouvelable tous les ans), la structure de la branche est assez bien connue. L'Agence fédérale pour l'emploi (Bundesagentur für Arbeit, Nuremberg), qui centralise le recensement des autorisations délivrées par ses directions régionales, fait ainsi état de 20800 entreprises en juin 2007 (dernier relevé en date, publié en février 2008). Ce nombre est en hausse de $21 \%$ en un an, et même de $138 \%$ depuis 1997, année où le régime des conditions d'emploi a été assoupli pour la

21000 entreprises en 2007, $138 \%$ de plus qu'en 1997 
Forte progression dans les régions en restructuration

Intérim : le " baromètre " du marché de l'emploi

La nomenclature classe l'intérim dans le tertiaire...

... mais la demande vient de l'industrie première fois : depuis, la conclusion d'un CDD avec l'entreprise d'accueil est possible sans justification, et les séries de missions ne sont plus limitées dans le temps. Au fil de la décennie, la plus forte augmentation a été constatée en Hesse (+281\%). Entre 2006 et 2007, la progression la plus dynamique (+54\%) a été enregistrée en Saxe-Anhalt et en Thuringe ; il est vrai que le salaire horaire est plus bas à l'est qu'à l'ouest.

L'Agence de Nuremberg recense 731000 travailleurs intérimaires en juin 2007, un nombre en hausse de $22 \%$ en un an et de $244 \%$ en dix ans. La distribution régionale des emplois a suivi la même évolution que celle des créations d'entreprises : la Hesse mène avec $+434 \%$ en dix ans. Mais la plus forte progression s'observe à l'est - la Saxe-Anhalt et la Thuringe ont connu depuis 2006 une augmentation de $35 \%$ - et, plus généralement, dans les régions en restructuration, donc aussi dans l'ouest du pays.

Dans la mesure où le secteur de l'intérim est considéré comme « le baromètre du marché de l'emploi » puisqu'il reflète fidèlement les tendances à venir dans les deux mois, et que les instituts économiques ont besoin de données à jour pour évaluer la demande à l'échelle macroéconomique, l'Institut der Deutschen Wirtschaft réalise depuis le début de 2006 des enquêtes mensuelles auprès de 1000 entreprises, en coopération avec la Fédération patronale Bundesverband Zeitarbeit (BZA) qui représente une partie des agences. Les chiffres publiés en avril 2008 portent à près de 750000 le nombre d'emplois enregistrés pour décembre 2007 (+19\% en un an). Les agences interrogées prévoient une hausse de 10,5\% au premier semestre 2008 (bza.de/649.html), ce qui signifie pour la conjoncture que « la demande de main d'œuvre reste soutenue » (iwd, 14/08).

La structure de l'emploi intérimaire par grandes catégories de métiers, telle que la présente l'Agence de Nuremberg - dont la vocation est le placement des chômeurs -, révèle une forte tendance à la tertiarisation en l'espace de dix ans. En juin 2007, $27 \%$ des intérimaires étaient employés dans les services $(20 \%$ en 1997), $24 \%$ dans la métallurgie et l'électrotechnique (contre $41 \%$ ), et $5 \%$ exerçaient un métier de technicien (3\%) ; $34 \%$ avaient une fonction d'auxiliaire (26\% en 1997), le restant appartenant à la catégorie « divers ».

Mais ce constat, qui influence les positions syndicales, est quelque peu faussé. D'abord, la production manufacturière est réduite ici à deux branches, certes de poids dans la structure économique de l'Allemagne : la métallurgie et l'électrotechnique (domaine d'IG Metall); mais la seule chute de leur part ne permet pas d'en conclure à une baisse du secondaire en général. Ensuite, le fait que, dans la nomenclature statistique, l'industrie n'est prise en considération que sous l'angle des activités de production réduit par ailleurs sa part dans la structure des activités ; or au fil des mutations de l'économie, nombre de fonctions qui relèvent aujourd'hui de la catégorie des services (comptabilité, logistique, $\mathrm{GRH} .$. ) avaient été externalisées par les entreprises industrielles (voir REA $58 / 02)$, elles restent toutefois dans le périmètre d'activités de ces dernières. Cela nourrit à l'arrière-plan la rivalité entre ver .di (services) et IG Metall sur le découpage de leur 'territoire'. Enfin, comme le souligne Destatis dans une étude sectorielle publiée en mars 2008 et réalisée sur la base du recensement Mikrozensus effectué en 2006, entreprises et employés de l'intérim «sont par définition affectés au secteur du tertiaire (services aux entreprises) " dans les statistiques. Mais pas dans la réalité syndicale et économique.

Car les résultats de ce Mikrozensus 2006, établies sur d'autres bases (enquêtes auprès de la population active) que les relevés statistiques, révèlent à l'inverse que près de $50 \%$ des intérimaires exercent une mission dans les métiers liés à la production manufacturière (22\% seulement du total des actifs) et que près de $45 \%$ seulement travaillent dans le tertiaire (contre $67 \%$ du total des actifs). "La comparaison avec la distribution de l'ensemble des actifs occupés sur les trois secteurs fait apparaître une part surdimensionnée du secondaire ». Soit dit en passant, ce constat contribue aussi à expliquer pourquoi la 
part des hommes atteint $66 \%$ dans l'intérim, alors qu'elle n'est que de $55 \%$ dans la population active. Mais il confirme surtout le rôle clef qui revient à l'intérim dans la flexibilité de l'industrie allemande.

La même étude révèle aussi que la part du temps partiel dans l'emploi intérimaire (moins de 151 heures par mois) est inférieure à celle qu'elle a dans la population active, avec $17 \%$ contre $26 \%$. Le temps plein est donc la norme. Quant à la part des CDD chez les employés des sociétés d'intérim (31\%), elle est deux fois plus élevée que dans l'ensemble des actifs (15\%), ce qui étaie le rôle de transition entre deux emplois fixes comme de retour en emploi que joue aussi le travail temporaire. Mais ces taux doivent être maniés avec prudence, rappelle Destatis, puisqu'ils reposent sur l'évaluation subjective de leur situation par les personnes interrogées dans le cadre du Mikrozensus. Cela étant, $20 \%$ des sondés étaient au chômage un an avant leur embauche par une société d'intérim.

Pour sa part, l'Agence fédérale pour l'emploi explique que, au cours du premier semestre 2007, $67 \%$ des contrats d'intérim ont été conclus avec des personnes "n'exerçant aucun emploi dans la période précédant immédiatement leur embauche". Et elle précise que "le travail temporaire offre une perspective aux chômeurs, aux salariés menacés de chômage, aux personnes en quête d'un premier emploi ou souhaitant retourner en emploi ». En chiffres : $48 \%$ de chômeurs de courte durée (moins d'un an), $13 \%$ de chômeurs de longue durée, $33 \%$ d'actifs occupés (contrat d'intérim conclu immédiatement après la cessation de l'activité précédente ; le tiers de cette cohorte travaillait déjà dans l'intérim) et $8 \%$ d'entrants.

Autrement dit, l'intérim allemand n'est pas ce tremplin d'insertion professionnelle qu'il est pour la plupart des jeunes Français, puisque les jeunes Allemands ont généralement acquis, durant leur formation duale, cette 'employabilité' qui fait défaut aux sortants du système français de formation générale (voir REA 69/04). D'autre part, l'intérim est choisi majoritairement par des actifs dont la qualification est en adéquation avec la demande des entreprises d'accueil et contribue de ce fait à la mobilité professionnelle. Cela explique a contrario pourquoi cette forme de travail n'absorbe que peu de chômeurs de longue durée ; leurs qualifications sont le plus souvent obsolètes.

L'intérim n'est donc pas non plus, outre-Rhin, le moyen le plus adapté à la réinsertion professionnelle des personnes les plus difficiles à placer, une mission confiée aux agences pour l'emploi allemandes qui l'exercent sous diverses formes dont le partenariat avec les société d'intérim dans le cadre des " agences de service de personnel » (Personal-Service-Agenturen, PSA) mises en place par les lois Hartz (voir REA 62/03). L'approche de ces dernières, comme du placement en général, est centrée sur le demandeur d'emploi, à l'opposé des agences d'intérim qui sont centrées, elles, sur la demande de main d'œuvre émanant des entreprises.

L'intérim, mieux à même de ce fait de satisfaire just in time la demande des entreprises, permet dès lors aux mieux qualifiés de (re)trouver rapidement un emploi dans un établissement d'accueil qui, souvent, les embauche à l'issue de leur mission. Le travail temporaire contribue ainsi à la fluidité du marché du travail, remplissant parfaitement l'un des objectifs de l'assouplissement de sa réglementation dans le cadre des réformes sociales du second gouvernement Schröder. La forte rotation des intérimaires en atteste : selon l'Agence de Nuremberg, la durée des contrats est inférieure à 3 mois dans $55 \%$ des cas. Cette fluidité de l'offre (nommée "précarité » dans la rhétorique syndicale) est adaptée à la demande : au début d'un cycle de croissance de leurs activités, les entreprises hésitent généralement à embaucher et préfèrent recourir aux heures supplémentaires en attendant que la croissance se confirme ; ensuite, elles couvrent leurs besoins accrus de main-d'œuvre par des missions d'intérim avant de se mettre à pratiquer de nouvelles embauches. Et c'est alors que, souvent, elles 'débauchent' leurs anciens intérimaires dont elles ont pu apprécier les compétences.
L'intérim pour la transition entre deux emplois

L'intérim contribue à la fluidité du marché du travail...

... grâce à l'adéquation entre offre et demande, ... 
... et il apporte une forme de «flexicurité »

La structure du travail temporaire par âges, telle que la révèle Destatis, confirme à sa manière cette adéquation entre offre et demande : c'est parmi les 30-44 ans que se recrute la plus grande partie des intérimaires, et la part de ceux-ci (43\%) est identique à celle de la même tranche d'âge dans la population active occupée. Le niveau de qualification constaté abonde dans le même sens : $64 \%$ des intérimaires ont un diplôme de fin de formation professionnelle (environ $55 \%$ seulement dans l'ensemble de la population active). II faut relever toutefois que $25 \%$ des intérimaires ne disposent pas d'un tel diplôme (contre $20 \%$ du total des actifs). Cela dit, presque tous les intérimaires (80\%) ont achevé leur formation scolaire générale, disposant soit du certificat de fin d'études de la Hauptschule, soit de l'équivalent allemand du BEPC (voir REA 75/06). Mais, comparés à l'ensemble de la population active, les intérimaires ont globalement un niveau de qualification inférieur, résume Destatis. Le travail temporaire contribue donc aussi à maintenir en emploi des actifs qui, du fait de la tendance générale à la hausse des qualifications, seraient sinon moins demandés par les entreprises. Dans le même temps, il contribue à une ouverture modérée de la fourchette salariale vers le bas, remplissant par là même un autre objectif lié à la réforme de sa réglementation dans le cadre de l'Agenda 2010.

Les agences d'intérim contribuent donc à la «flexicurité », dans la définition qu'en donnait V. Spidla, Commissaire européen en charge de l'Emploi, dans un discours tenu en 2006 à l'OIT : "sécurité des travailleurs tout au long de diverses périodes d'emploi » (euro-ciett.org). Et pour les entreprises, elles constituent une alternative attractive au réseau public de placement dans la mesure où elles leur offrent leurs prestations pour le recrutement et la gestion d'une gamme diversifiée de main-d'œuvre, tant qualifiée que non qualifiée, la première catégorie gagnant en importance dans le contexte de la pénurie actuelle de main-d'œuvre hautement qualifiée (ingénieurs et techniciens).

\section{L'évolution de la réglementation du travail temporaire et des lignes d'affrontement sous-jacentes}

Depuis sa légalisation en 1972, l'intérim a connu plusieurs modifications de sa réglementation. Dans la période précédant 2003, il est strictement encadré. Les syndicats du DGB, faute de pouvoir obtenir son interdiction, cherchent à lui appliquer ou faire appliquer un statut le plus proche possible de l'emploi régulier. Or après quelques avancées en matière d'organisation du secteur, et confrontés à une progression incompressible du chômage à la fin des années 1990, ils changent de perspective et le considèrent comme un instrument de la politique active pour l'emploi. Leur position reste néanmoins ambiguë: s'ils acceptent le caractère transitoire du travail temporaire, ils cherchent à le limiter le plus possible pour 'normaliser' ce type d'emploi atypique en tentant d'instaurer, par divers biais législatifs, une égalité de traitement avec l'emploi régulier.

A partir de 2003, dans le cadre des réformes sociales des gouvernements Schröder, dont les « lois Hartz », le régime de l'intérim est considérablement assoupli et devient l'un des instruments de la nouvelle politique de flexibilisation de l'emploi au côté d'un régime moins strict de protection contre le licenciement. Si les syndicats du DGB, forts des négociations en cours sur un projet de Directive sur l'intérim, n'ont pas abandonné l'objectif de l'égalité de traitement, prévu désormais par la version amendée de la loi allemande sur le travail temporaire, ils n'en préfèrent pas moins dans un premier temps recourir à la méthode contractuelle et concluent des accords de branche, espérant ainsi organiser le secteur.

\section{2-2002 : légalisation, puis intégration dans la politique pour l'emploi}

1972 : le droit du travail s'applique à l'intérim
La Loi sur la cession des salariés (Arbeitnehmerüberlassungsgesetz : AÜG) du 07-08-1972 pose le cadre réglementaire d'une profession considérée jusque-là comme illégale, mais non moins florissante. Elle avait été adoptée à la suite 
d'un arrêt rendu en 1967 par le Tribunal constitutionnel fédéral qui avait été saisi par l'Office fédéral du Travail (rebaptisé depuis Agence fédérale pour l'emploi) pour concurrence illicite. De surcroît, le statut des intérimaires n'était en rien réglementé, ni en termes de droit du travail, ni en matière de protection sociale, ce qui avait valu aux agences d'intérim le surnom de "marchands d'esclaves » et terni pendant longtemps l'image de la profession.

La loi de 1972 soumet donc l'intérim aux règles générales du droit du travail, tout en lui aménageant un régime spécifique, strictement encadré : elle interdit notamment les contrats de CDD avec l'agence d'intérim et l'enchaînement des missions dans une même entreprise d'accueil, et elle limite la durée des missions. Son approche vise d'une part à faire prendre en charge par l'agence le risque lié aux périodes d'inactivité de leur employé (via la conclusion de CDI) et, d'autre part, à empêcher dans l'entreprise d'accueil le remplacement d'un salarié fixe par un intérimaire. Les syndicats du DGB n'en restent pas moins farouchement opposés au travail temporaire du fait de la précarité inhérente à cette forme d'activité, réclamant à plusieurs reprises son interdiction. Pour protéger les salariés allemands du BTP de la concurrence étrangère, un amendement à la loi interdit l'intérim dans ce secteur ; ce traitement particulier débouchera plus tard sur un régime spécifique, adopté en préfiguration dans le droit allemand de la Directive sur les travailleurs détachés, et sur l'instauration d'un SMIC légal dans le BTP.

Confronté à une montée du chômage dont on perçoit dès l'époque qu'il est en partie nourri par les rigidités du droit du travail, surtout en matière de licenciement, le gouvernement fédéral modifie une nouvelle fois la loi en 1985, pour allonger la durée maximale des missions dans le but de flexibiliser la réglementation du travail, puis une nouvelle fois encore en 1994, année où est levée l'interdiction d'enchaîner les missions pour les chômeurs difficiles à placer. Ces assouplissements favorisent la croissance de l'intérim et, sur fond de montée du chômage, forcent la différenciation salariale entre l'emploi régulier et temporaire. Malgré cette tendance et les abus constatés, et au nom de l'intérêt supérieur de la lutte contre le chômage, le DGB supprime dans son programme fondamental adopté en 1996 la revendication d'une interdiction de l'intérim.

De nouveaux assouplissements sont apportés en 1997 : la durée des missions et des enchaînements est encore allongée et, surtout, les contrats de CDD sont désormais autorisés sans qu'il ne soit plus nécessaire d'en justifier la limitation dans le temps ; cette disposition ne s'applique toutefois qu'à un seul CDD par employé. Parallèlement et indépendamment de la question du travail temporaire, la Loi de protection contre les licenciements (Kündigungsschutzgesetz) de 1969 est assouplie dès l'arrivée au pouvoir du gouvernement Schröder en 1998 pour préserver l'emploi : le seuil d'application de la loi est abaissé de plus de 10 à plus de 5 salariés (il sera rétabli en 2004 ; Zumfelde, 2005).

A la fin des années 1990, quelques conventions collectives sont conclues dans le secteur à l'échelon régional, incluant notamment la constitution de conseils d'entreprise au sein des sociétés d'intérim. Les deux parties en tirent bénéfice : les syndicats posent les jalons de l'organisation de ce qui préfigure une branche, et les fédérations des agences d'intérim se voient ainsi faciliter l'accès aux entreprises d'accueil où règne un fort taux d'organisation syndicale. A cette époque s'opère un changement général de paradigme : l'intérim est désormais considéré comme un instrument de la politique pour l'emploi.

Au début du millénaire, toujours sous le premier gouvernement Schröder, trois lois sont adoptées qui modifient indirectement l'AÜG. La Loi du 23-07-2001 (Gesetz zur Reform des Betriebsverfassungsgesetzes), portant réforme de la Loi sur la Constitution interne de l'entreprise de 1972, étend aux intérimaires le droit de vote au conseil d'entreprise de l'établissement d'accueil dès lors que leur mission dépasse 3 mois; elle leur ouvre également le droit de vote aux élections du représentant des salariés au conseil de surveillance (Lattard, 2001). La loi du 10-12-2001 relative à la réforme du dispositif de la politique
Le DGB reste opposé à cette forme légale de "précarité »

Après 1994 : priorité à l'emploi et levée d'une partie des rigidités

L'intérim devient un instrument de la politique pour l'emploi

Egalité de traitement en contrepartie des allégements 
Commission Hartz et flexibilisation du marché du travail

Loi Hartz I : application du principe de non-discrimination s'il n'y a pas de convention tarifaire

Les fédérations patronales déboutées sur un régime spécial des CDD dans l'intérim... pour l'emploi (Gesetz zur Reform der arbeitsmarktpolitischen Instrumente, communément appelée Loi Job-AQTIV) remonte à deux ans la durée maximale d'une mission et fait obligation à l'entreprise d'accueil d'appliquer à l'intérimaire, dès le $13^{\mathrm{e}}$ mois de sa mission, les conditions de travail et la grille salariale en vigueur chez elle (principe de l'égalité de traitement). Enfin, la loi du 23-07-2002 facilitant la lutte contre le travail illégal (Gesetz zur Erleichterung der Bekämpfung von illegaler Beschäftigung und Schwarzarbeit) supprime le délai de 30 jours ouvrables au-delà duquel la mise à disposition d'un travailleur étranger dans des conditions de travail et de salaire discriminatoires par rapport au régime applicable aux nationaux constitue un délit.

\section{Depuis 2003 : régime assoupli pour un outil de flexibilisation de l'emploi}

A la fin du premier mandat de G. Schröder, le nombre de chômeurs franchit la barre des 4 millions. Dans ce contexte (et sur fond d'un scandale à l'Office fédéral du travail, accusé de maquiller son taux de placement), le gouvernement fédéral institue une Commission pour des services modernes sur le marché de l'emploi (Kommission für moderne Dienstleistungen am Arbeitsmarkt), chargée d'élaborer un train de mesures de lutte contre le chômage. Cette «Commission Hartz », dans laquelle siégeaient des représentants des syndicats IG Metall et ver.di, développe un nouveau paradigme pour le travail temporaire. Désormais, celui-ci est considéré comme un vecteur de flexibilisation du marché du travail.

A la suite des travaux de la commission, toute une série de mesures sont prises, dont une réforme de l'Office de Nuremberg (rebaptisé Agence pour l'emploi à cette occasion), la création des PSA ou l'assouplissement du régime des CDD et des petits boulots (dont les mini-jobs). Dès sa reconduction à l'automne 2002, le gouvernement Schröder met en œuvre les recommandations de la commission : les « Lois Hartz » entreront en vigueur progressivement.

Le 23-12-2002 est votée la Première loi pour des services modernes sur le marché de l'emploi (Erstes Gesetz für moderne Dienstleistungen am Arbeitsmarkt), plus connue sous le nom de «Loi Hartz I » et entrée en vigueur le 01-01-2003. En ce qui concerne l'intérim, elle lève toutes les restrictions propres au secteur (dont l'interdiction concernant le BTP), concrétisant ainsi le changement de paradigme adopté lors des travaux de la commission, tout en intégrant le principe clef du projet de Directive sur l'intérim en cours de négociation à l'échelon communautaire, principe que revendiquent également les syndicats du DGB.

En contrepartie de la déréglementation du travail temporaire, elle généralise donc le principe de non-discrimination sous la forme de l'égalité de traitement (conditions de travail et salaires) entre travailleurs intérimaires et salariés fixes de l'entreprise d'accueil. Mais à une condition: que les partenaires sociaux n'aient pas conclu de conventions collectives propres au travail temporaire. La «Loi Hartz III » du 23-12-2003 complète la première en instaurant, pour l'agence d'intérim comme pour l'intérimaire, un droit d'information sur les conditions de travail en vigueur dans l'entreprise d'accueil quand aucune convention tarifaire ne s'applique à elle. Lorsqu'il en existe une, le fait que les conventions tarifaires soient publiées rend en effet caduc un tel droit.

Le mouvement patronal salue cette déréglementation, en attendant une embellie sur le marché de l'emploi en général. Néanmoins, les fédérations de l'intérim plaident pour que le régime des CDD conclus avec leurs employés soit spécifié en ce sens que devrait être créé par la loi un motif ad hoc justifiant le caractère temporaire du contrat ; le droit commun du travail fait en effet obligation de motiver expressément tout CDD. Le gouvernement fédéral s'oppose à cette revendication, se basant sur la juridiction du Tribunal fédéral du Travail. Celui-ci considère en effet que "l'incertitude sur les besoins futurs en maind'œuvre ne suffit pas à légitimer objectivement la limitation de la durée d'un contrat de travail ; en effet, cette incertitude fait partie intégrante du risque entrepreneurial et ne peut donc être répercutée sur les salariés via la conclusion 
de contrats en CDD ", résume le gouvernement (BT-Drucksache 15/6008). On le voit, même si le travail temporaire est dans l'ensemble 'normalisé', il conserve le statut d'exception d'une forme de travail 'atypique'.

Les fédérations de l'intérim s'insurgent aussi contre l'égalité de traitement. Elles saisissent le Tribunal constitutionnel fédéral, invoquant la violation de l'art. $9 \S 3$ de la Loi fondamentale qui garantit la liberté de coalition - le principe qui fonde l'autonomie des partenaires sociaux en matière de régulation sociale. Le 29-122004, la Cour suprême rejette leur recours, considérant qu'il n'a aucune chance d'aboutir. Le pivot de son argumentation est le suivant : la protection des employés de l'intérim étant suffisamment garantie par les nouvelles dispositions légales, et l'objectif de celles-ci étant de créer des passerelles entre chômage et emploi, elles servent l'intérêt général et sont donc conformes à la Constitution.

De leur côté, les syndicats du DGB saluent la nouvelle réglementation. S'ils ont une préférence pour l'égalité de traitement, la solution d'une convention de branche les satisfait totalement, puisqu'elle leur offre la possibilité d'organiser le secteur de l'intérim. L'option d'une régulation contractuelle de branche ayant elle aussi la préférence du législateur puisqu'elle est la règle en Allemagne, les modifications afférentes apportées à la Loi $A \ddot{U G}$ en 2002 n'entrent en vigueur que le 01-01-2004 pour laisser aux partenaires sociaux le temps de négocier. Pour éviter que le législateur n'ait à intervenir (et pour éviter l'obligation d'égalité de traitement), le patronat s'organise. Avant la fin 2003, les partenaires sociaux sont constitués, et plusieurs conventions de branche sont conclues, à l'échelle de l'Allemagne, des régions ou dans des entreprises. Elles prévoient alors des salaires planchers s'échelonnant entre 5,60€ et 6,80€ par heure, les salaires plafonds variant entre $14,17 €$ et $16,28 €$ selon que les établissements d'accueil sont à l'est ou à l'ouest. Deux de ces conventions (nationales) sont au cœur des enjeux depuis leur signature.

La première a été signée, au niveau patronal, par deux fédérations de l'intérim : la Bundesverband Zeitarbeit Personal-Dienstleistungen (BZA) et I'Interessenverband Deutscher Zeitarbeitsunternehmen (IGZ). Leurs partenaires syndicaux, rassemblés pour l'occasion en un groupement tarifaire, sont membres de la Confédération DGB. II s'agit de : IG BCE (chimie), IG Metall (travail des métaux), IG Bau (BTP, agriculture), NGG (gastronomie/hôtellerie), GEW (éducation et recherche), TRANSNET (transports) et GdP (police).

La seconde a été signée, d'un côté, par la Fédération des PME de l'intérim, la Mittelstandsvereinigung Zeitarbeit (MVZ), ainsi que celle des agences du nord de la Bavière, l'Interessengemeinschaft Nordbayerischer Zeitarbeitsunternehmen (INZ) qui a depuis fusionné avec la première, rebaptisée à cette occasion Arbeitgeberverband Mittelständischer Personaldienstleister (AMP). De l'autre, les signataires sont associés en groupement ad hoc comme les organisations du DGB, mais leurs membres appartiennent à la Fédération des syndicats chrétiens, le Christlicher Gewerkschaftsbund (CGB). II s'agit des syndicats suivants: CGM (travail des métaux), DHV (industrie et commerce), GÖD (services publics), Union Ganymed (gastronomie et hôtellerie) et VDT (ingénieurs et techniciens).

Dès leur signature, ces conventions sont sources de conflit, puisque les organisations du DGB se voient là exposées à une concurrence qu'elles considèrent non seulement comme "déloyale », mais bien plus encore "illégale », et qu'elles vont chercher à évincer, en vain jusqu'ici, par une politique systématique de recours devant les tribunaux. Le motif de leurs saisines est le même depuis de longues années, dans l'intérim comme dans d'autres branches (dont la métallurgie) : l'absence de capacité à contracter des syndicats chrétiens, du fait d'une représentativité jugée trop faible, voire inexistante. Néanmoins, dans une affaire opposant IG Metall et son concurrent Christliche Gewerkschaft Metall (CGM), le Tribunal fédéral du travail a rendu le 28 mars 2006 un arrêt dans lequel il considère que le CGM remplit toutes les conditions requises par le statut de syndicat : une capacité suffisante pour faire valoir les intérêts des salariés face à ses
... et sur le principe d'égalité de traitement

Législateur, patronat et syndicats préfèrent la contractualisation

Le DGB, en concurrence...

... avec les syndicats chrétiens,...

... saisit les tribunaux pour leur dénier toute représentativité 
homologues patronaux. Peu importe en effet sa faible représentativité quantitative (en l'occurrence $2 \%$ des salariés dans le travail des métaux), celle-ci étant largement compensée par sa capacité à organiser un secteur, ce dont attestent quelque 550 conventions conclues dans la branche considérée (réf. 1 ABR 58/04). Mais la jurisprudence est loin d'être univoque, chacun des syndicats du DGB cherchant à endiguer la concurrence chrétienne avec plus ou moins de succès. Et tant que le Tribunal constitutionnel fédéral n'aura pas statué sur le fond (principe de la liberté d'association), la guerre intestine entre les deux confédérations DGB et CGB, avivée par les mutations de l'économie et, en ce qui concerne l'intérim, par l'accélération de l'outsourcing, ne sera pas pacifiée.

En avril 2008, le conflit s'est envenimé : IG Metall (rappelons qu'une grande partie des intérimaires est occupée dans la métallurgie) a lancé une véritable 'guerilla' auprès des entreprises d'accueil affiliées à la fédération patronale chrétienne AMP, leur envoyant un formulaire de " contrat de loyauté " à signer avant le $1^{\mathrm{er}}$ mai et accompagné d'une mise en demeure. Ce «contrat » vise à leur faire adopter les termes des conventions conclues par les membres du DGB (et dont les salaires planchers sont supérieurs de 30 à 50 cents à ceux de la 'concurrence'), voire à leur faire conclure avec les établissements d'accueil des conventions introduisant l'égalité de traitement. Gesamtmetall, partenaire patronal d'IG Metall, s'insurge contre de telles pratiques qu'il juge anti-concurrentielles. Quant à la fédération AMP (partenaire du CGB), elle a annoncé qu'elle allait saisir l'Office fédéral des Cartels...

\section{SMIC légal et droit des cartels pour réguler à l'avenir le marché du travail ?}

La branche n'est plus la référence pour la régulation sociale

Elle a perdu ce statut au fil des mutations économiques et sociales

Les conventions de branche ne s'appliquent plus qu'à la moitié des entreprises
Ce paroxysme de violence, récent en Allemagne et surtout inhabituel de la part d'une organisation aussi établie qu'IG Metall, révèle que le "monopole bilatéral » du partenariat social (Haucap, 2008), reposant traditionnellement sur le couple constitué par une fédération patronale de branche et un syndicat unitaire de branche (par exemple Gesamtmetall/IG Metall) n'est plus la règle. La branche est en train de perdre son statut de référence pour la régulation des relations sociales (Lattard, 2005). Et, la concurrence inter-syndicale tendant à se généraliser, "le modèle syndical allemand n'existe plus » (Schrœder, 2005).

Cette évolution s'explique par les mutations des activités qui ont brouillé le périmètre des branches et en ont généré de nouvelles, non ou faiblement organisées, mais aussi sous l'effet d'un corporatisme menant à des conditions-cadre de plus en plus rigides, incitant nombre d'entreprises à ne pas s'affilier à une fédération de branche, ce qui les place hors du champ d'application des conventions. Cette dernière tendance a pris une grande ampleur peu après l'unification, dans les nouveaux Länder où avait été conclu un rattrapage salarial disproportionné par rapport à la productivité (Schrœder, 2005). Elle s'est étendue à l'ouest, l'externalisation des activités favorisant cette désaffection. Les syndicats ont certes tenté de l'endiguer, notamment en multipliant les clauses dérogatoires, mais en vain. Dans le même temps se multipliaient dans le tertiaire les représentations professionnelles ou catégorielles, comme les organisations Cockpit (pilotes) ou GDL (conducteurs de train), cette tendance apportant elle aussi sa contribution à l'éclatement des branches. Enfin, les mutations de la société, et notamment l'individualisation des comportements, favorisent de leur côté une baisse du taux de syndicalisation chez les salariés.

La fusion, en 2001, de plusieurs syndicats des services au sein d'un conglomérat rassemblant quelque 1000 métiers : ver.di, a été une tentative de réponse institutionnelle à ces évolutions. Mais ce nouveau géant n'est jamais parvenu à organiser le tertiaire selon la logique traditionnelle de branche. Et, comme IG Metall, ancêtre du modèle allemand du syndicat unitaire de branche, il souffre d'une énorme perte de représentativité. Certes, ces deux principales organisa- 
tions au sein du DGB comptent chacune encore environ 2,3 millions d'adhérents selon les estimations, mais ne communiquent plus guère sur ces chiffres, ce qui est révélateur de la crise qu'ils traversent. De 1999 à 2006, le DGB en tout cas a perdu $18 \%$ de ses adhérents, leur nombre tombant à 6,59 millions (Haucap, 2008). Le patronat, en face, subissait une désaffection comparable (néanmoins impossible à chiffrer). L'affaiblissement du partenariat social a abouti au fait que, en moins de dix ans, de 1998 à 2006, la part des entreprises liées par des conventions tarifaires de branche est tombée de $53 \%$ à $40 \%$ à l'ouest (de $33 \%$ à $24 \%$ à l'est). Quant au taux de salariés ainsi protégés, il est tombé de $76 \%$ à $65 \%$ à l'ouest, de $63 \%$ à $54 \%$ à l'est (Bispinck, 2008).

La crise du modèle de la convention tarifaire de branche est particulièrement flagrante dans l'intérim, un secteur multi-positionné par nature. IG Metall, puissant dans les secteurs clefs de l'industrie allemande, est concerné en premier chef puisque c'est dans sa 'branche' que se recrutent le plus d'intérimaires. Mais il n'est jamais parvenu à les organiser réellement, ni dans les agences d'intérim ni dans les entreprises d'accueil, aussi parce que, facteur aggravant, ce "déficit d'organisation est directement lié à la structure même du contrat d'intérim » (Wölfle, 2008). Or la stratégie de 'normalisation' tentée par IG Metall a été mise à mal par les syndicats chrétiens, mieux représentés dans le travail temporaire qu'ailleurs, ce qui explique la virulence particulière de l'offensive menée par un syndicat aux abois face à l'émergence de ce concurrent.

La stratégie avait souffert aussi, préalablement, de la concurrence de ver.di qui, arguant de l'appartenance statistique de l'intérim au tertiaire, s'estimait de jure le seul compétent. Mais encore moins que son homologue de la métallurgie, le géant des services n'était parvenu à organiser l'intérim. Ni d'autres 'branches' d'ailleurs, sauf dans les services publics en voie de libéralisation comme les services postaux dont il a fait son territoire réservé. Ver.di avait dès lors opté très tôt pour une autre stratégie dont il peut être considéré comme le spécialiste depuis son succès à large échelle dans le secteur postal (introduction d'un SMIC légal, voir REA 85/08) : le "campaigning » ou la politisation d'un thème porteur dans l'espace public, en l'occurrence la « précarisation ».

Son exemple est aujourd'hui suivi par IG Metall, après le constat d'échec d'une autre tentative, plus institutionnelle, d'organisation par le développement de structures participatives. En novembre 2004 avait été créé à cet effet un réseau virtuel, "ZeitarbeiterInnen - Ohne Organisation Machtlos » (ZOOM) : "Intérimaires - impuissants si non organisé(e)s ». La plateforme Internet igmetallzoom.de, qui est toujours active, offre un forum de discussion et de libre expression, met à disposition une documentation relative à la réglementation dans l'intérim (conventions collectives ou d'entreprise, jurisprudence, etc.), délivre des conseils en cas de licenciement et informe, de manière générale, sur le droit des salariés et leur représentation via les conseils d'entreprise. Elle n'a guère rempli son objectif : le recrutement d'adhérents.

\section{L'intérim, surface de projection pour un nouveau modèle social}

L'intérim, structurellement hors du champ du partenariat de branche, se trouve ainsi propulsé au rôle d'enjeu clef dans une série de débats sur l'avenir de la régulation sociale en Allemagne. La revendication d'un régime spécifique pour cette profession masque en réalité la montée en puissance d'une attitude plus défensive que proactive face aux profondes évolutions structurelles en cours, à l'échelon de l'Allemagne comme à celui de l'Europe. II est vrai aussi que le travail temporaire offre une surface de projection idéale pour l'esquisse d'un 'nouveau' modèle social allemand. Et que, avec son image jamais totalement effacée de " marchands d'esclaves ", il offre un support particulièrement bien adapté à une stratégie de politisation qui vise, par-delà la lutte contre le « dumping salarial » ou la protection des moins qualifiés, à mettre fin à la concurrence inter-syndicale par un appel au législateur - au nom d'un intérêt social supérieur.
IG Metall n'a pas réussi à organiser l'intérim et se trouve aux abois

Comme ver.di, il recourt désormais au campaigning... ... après l'échec d'une
stratégie participative

Appel au législateur au nom d'un intérêt social supérieur 
Objectif : un monopole salarial consacré par la loi

Le législateur ne peut intervenir en dernier recours que dans trois cas

La procédure d'extension n'est pas envisageable

Une seule solution : la lo sur les travailleurs détachés

Coalition d'intérêts entre DGB et géants de l'intérim...
L'introduction d'un SMIC légal dans le secteur postal crée un précédent. La revendication d'un minimum fixé par la loi est favorisée par le fait que le salaire plancher (un minimum auquel il ne peut être dérogé) fixé par une convention tarifaire s'applique exclusivement aux parties contractantes. Les entreprises et salariés hors du champ de cette convention ne sont donc pas couverts, ce qui est un des facteurs d'ouverture de l'éventail des salaires vers le bas du fait de la baisse générale du taux d'organisation. Or dans l'intérim, il existe deux principales conventions concurrentes, celle des syndicats du DGB présentant en l'occurrence les salaires les plus élevés. La 'légalisation' de ces planchers mettrait fin à la concurrence en instaurant une norme salariale unique. La revendication d'un SMIC légal dans l'intérim va au-delà du principe d'égalité de traitement inscrit dans le droit allemand. Mais il y figure en dernier recours seulement, c'est-à-dire en cas de défaillance des partenaires sociaux. Or ceux-ci ont signé des conventions couvrant la quasi-totalité du secteur, ce qui rend ce principe inapplicable.

Reste donc à trouver un autre biais. II n'y a que trois possibilités, le législateur allemand ne pouvant intervenir que si l'intérêt général justifie une entorse à l'autonomie tarifaire des partenaires sociaux que garantit la Loi fondamentale. La première est la Loi de 1952 sur les conditions de travail minimales (Mindestarbeitsbedingungsgesetz) qui permet au législateur de se substituer à des partenaires sociaux défaillants. Cette situation ne s'étant jamais présentée, la loi n'a jamais été appliquée (mais un projet de réforme existe).

La plus ancienne des deux autres possibilités est le recours à la procédure d'extension (Allgemeinverbindlichkeitserklärung) prévue par la Loi sur les conventions tarifaires (Tarifvertragsgesetz). Elle habilite le ministère fédéral du Travail à donner par décret force de loi sur l'ensemble du territoire à une convention - à condition que celle-ci lie au moins $50 \%$ des salariés de la branche, et que son extension se justifie par une situation "d'urgence sociale ». Elle est très peu appliquée (pour largement moins de $1 \%$ des conventions), et ne concerne guère actuellement que quelques secteurs isolés comme la coiffure ou la sécurité. Si l'urgence se laisse aisément démontrer par des études ad hoc comme ce «Rapport social sur l'intérim » (Sozialreport Zeitarbeit, 2007) d'IG Metall, le fait qu'il y ait deux conventions concurrentes dans l'intérim interdit l'application de cette procédure. La situation est la même que dans le secteur postal, où la procédure d'extension fut pourtant utilisée dans une première étape, au bénéfice de la convention la plus favorable (mais le Tribunal administratif de Berlin a invalidé cette procédure le 7 mars 2008).

La troisième possibilité est Loi Arbeitnehmer-Entsendegesetz (AEntG) du 2602-1996, votée dans le contexte de la Directive 71/96/CE sur les travailleurs détachés. Fondée sur le principe du pays d'accueil, elle vise à appliquer aux étrangers détachés en Allemagne les mêmes conditions de travail (et de salaire) qu'aux nationaux (en contrat fixe). Or cette loi avait été adoptée d'abord pour protéger les salariés du BTP est-allemand contre les concurrents esteuropéens grâce à la création d'un SMIC légal, étendu en 2006 à l'ensemble du BTP comme au traitement des façades. C'est cette loi qui avait permis l'adoption du SMIC postal (entre 8 et 9,80 € selon l'activité et sa localisation dans les Länder de l'est ou de l'ouest) après extension de son champ d'application à ce secteur, une fois force de loi accordée à la convention Ver.di/Deutsche Post. C'est la même combinaison de cette loi avec la procédure d'extension qui doit permettre l'instauration d'un SMIC légal dans l'intérim et sept autres secteurs, dont les services de dépendance, la formation continue ou les services de sécurité - autant de secteurs pour lesquels ne s'applique pas la Directive services (régie par le principe du pays d'origine). Le ministère fédéral du Travail avait lancé au début de l'année un appel d'offres, à destination des "branches" candidates à être inscrites dans le champ d'application de la loi AEntG.

Pour l'intérim, la requête a été déposée par le couple DGB/BZA et IGZ, ces deux fédérations patronales réunissant essentiellement les géants du secteur, dont Adecco, Randstad, Persona Service, Manpower et Hays, pour ne citer que 
les cinq principaux. Elles avaient conclu en 2006, en prévision de cet appel d'offres, une convention destinée à être appliquée à l'ensemble de la 'branche' via la procédure d'extension couplée à la loi $A E n t G$, et fixant un salaire minimum unique dès 2008 , de $7,31 €$ à l'ouest et $6,36 €$ à l'est. Cette convention s'appliquerait donc aussi à la multitude de PME, représentées par l'AMP, partenaire des syndicats chrétiens. Or de tels seuils - destinés, selon la procédure envisagée, à interdire tout « dumping salarial » - sont de nature à pénaliser les petites agences, forcées de servir une demande plus indifférenciée que les grands groupes qui ont la taille requise pour se spécialiser dans les niches à fort potentiel : la demande d'intérimaires hautement qualifiés (Adecco vient par exemple de racheter le spécialiste DIS). L'écart entre ces deux catégories risque de se creuser d'autant plus que seuls les géants ont la dimension nécessaire pour servir un marché communautaire de plus en plus intégré, ce qui leur permet aussi de mettre à profit le différentiel salarial existant au sein de l'UE... La coalition d'intérêts entre membres du DGB et magnats de la branche nourrit donc aussi, à l'arrière-plan, la revendication d'un SMIC légal.

Un SMIC dans l'intérim, projeté officiellement selon une approche de redistribution, se solderait par une distorsion de la concurrence, dommageable in fine à l'emploi. La procédure retenue dans le cas de l'intérim comme des services postaux traduit clairement "l'option stratégique d'un abus de la loi AEntG dans un objectif d'entrave à la concurrence " (Haucap, 2008). Introduire un seuil salarial légal élevé dans l'intérim vise non seulement l'éviction des organisations patronales et syndicales concurrentes, mais crée aussi des barrières élevées à l'entrée du marché. La multiplication récente du recours au facteur salaire pour 'réguler' un marché, se surcroît par l'intervention de l'Etat, pose clairement aujourd'hui la question de l'articulation entre droit du travail (conventions salariales) et droit de la concurrence. Cela amène la Commission des monopoles, dont J. Haucap est membre, à plaider pour que, à l'avenir et le cas échéant, tout projet de fixation de salaires par voie législative soit soumis à l'avis préalable de l'Office fédéral des Cartels. Cela est d'autant plus justifié que jamais, au fil de l'histoire sociale de la RFA, l'articulation entre ces deux volets de l'ordre économique et social, n'a été réglée. Or, l'évolution révèle aujourd'hui " la perméabilité entre droit tarifaire et droit des cartels » (Haucap, 2008).

Le SMIC LEGAL DANS L'INTERIM N'EST ENCORE QU'UN PROJET, et il est vivement critiqué sous divers angles, y compris au sein même du gouvernement fédéral où s'affrontent les deux formations SPD et CDU/CSU, bien que la commission de coalition ait donné suite à une revendication conjointe du SPD et du DGB le 18 juin 2007. Il avait été convenu à cette occasion d'étendre le domaine d'application de la Loi sur les travailleurs détachés et de procéder à une révision de la Loi de 1952 sur les conditions de travail minimales afin de pouvoir instaurer des SMIC légaux dans tous les secteurs non régulés par voie contractuelle. Cette dernière mesure constitue également l'objectif ultime des syndicats du $\mathrm{DGB}$, qui prônent un salaire horaire minimum généralisé de 7,50€. Ce montant a déclenché une violente polémique à la fois sur le montant préconisé (les voix les plus modérées plaident pour l'abaisser à $4,50 €$ ) et sur le fait même qu'un salaire puisse être fixé par l'Etat.

Dans ce contexte, le projet de SMIC dans l'intérim prend une double fonction de test et d'expédient. La justification de la procédure retenue pour l'introduire est en effet peu tenable du point de vue juridique: l'intérim ne concerne en rien le détachement des travailleurs au sein de l'UE ; aucune concurrence « déloyale » n'est à attendre par ailleurs à partir de 2011 de la libre circulation des ressortissants est-européens ni de la prochaine mobilité des services au sein de l'UE. Ce ne sont là que des prétextes nourris de crispations protectionnistes et de visées électoralistes. Ils sont révélateurs de la profonde crise que traverse le syndicalisme social-démocrate et, plus généralement, un modèle social allemand né dans la société industrielle d'après-guerre et défié aujourd'hui par l'accélération 
du processus d'intégration européenne. Le choix de la procédure est en réalité un pis aller pour parer à une incapacité grandissante du partenariat social à répondre à la mission que lui confie la Constitution : la régulation des conditions de travail et des salaires. Son émiettement croissant amène fédérations patronales et syndicales à se tourner vers le législateur pour que celui-ci réglemente par la loi ce qui semble de plus en plus difficile à réguler par le contrat.

L'appel à la centralisation aux mains de l'Etat des décisions en matière de conditions de travail et de salaire, s'il débouche, comme le réclament le SPD et le DGB, sur l'adoption d'un SMIC généralisé pour l'ensemble de l'économie, ruinerait les fondements mêmes du contrat social allemand établi sur le principe de subsidiarité, c'est-à-dire l'autonomie des acteurs économiques et sociaux. C'est là la raison profonde qui a motivé un farouche appel conjoint des instituts de conjoncture et de l'Institut der Deutschen Wirtschaft, publié le 12 mars 2008 et intitulé : "Des chances pour l'emploi plutôt qu'un SMIC!" (Beschäftigungschancen statt Mindestlohn!). Un tel minimum légal, surtout s'il était fixé aux $7,50 €$ réclamés par les syndicats du DGB, serait catastrophique non seulement pour l'activité, mais encore plus pour l'emploi, expliquent les signataires qui citent expressément comme contre-exemple la France et ses barrières élevées à l'entrée sur le marché du travail pour les jeunes et les moins qualifiés. Ceux à qui pourtant, en Allemagne plus qu'en France, profite le travail temporaire puisqu'il leur donne accès à un emploi régulier. Toute l'ambiguïté de l'intérim est là, dans sa double nature d'instrument de lutte contre le chômage et de réserve de flexibilité pour l'économie. Le pénaliser sous prétexte de justice sociale n'est pas pour autant un moyen de résoudre ce double défi.

\section{Indications bibliographiques}

BISPINCK R., "Das deutsche Tarifmodell im Umbruch », in Auflösung des deutschen Tarifmodells?, Dossier ,Zeitgespräch', ZBW, Wirtschaftsdienst, 1/2008

BOURGEOIS I., "Marché postal allemand : libéralisation sans concurrence », Regards sur l'économie allemande, 85/2008

BUNDESAGENTUR FüR ARBEIT, Zeitarbeit. Branchen und Berufe in Deutschland, 19972007, février 2008 (www.statistik.arbeitsagentur.de)

DESTATIS, Zeitarbeit. Ergebnisse des Mikrozensus, mars 2008 (www.destatis.de)

HAUCAP J., "Erosion des Flächentarifsystems macht eine Wettbewerbsaufsicht auf dem Arbeitsmarkt notwendig », DIW-Wochenbericht, $n^{\circ} 6 / 2008$

HAUCAP J. et al., " Das deutsche Tarifkartell : Entstehung, Stabilität und aktuelle Reformvorschläge aus Sicht der Wettbewerbstheorie ", in OHR R. (ed), Arbeitsmarkt und Beschäftigung, Berlin, 2007

IG METALL, Sozialreport Zeitarbeit, mars 2007

LASSERRE R., "Les origines de la ,Sozialpartnerschaft' », in KREBS G. (ed), L'Allemagne de Konrad Adenauer, Asnières, 1982 (www.cirac.u-cergy.fr/workingpapers.php)

LATTARD A., "Négociation collective : quel avenir pour la convention de branche ? ", in BouRgeols I. (dir.), Le modèle social allemand en mutation, Cergy-Pontoise, 2005, et "La réforme de la 'Constitution interne de l'entreprise' en Allemagne ", Regards sur l'économie allemande, $n^{\circ} 50 / 01$

OCHEL W., "Tarifliche Mindestlöhne, Allgemeinverbindlichkeit und Entsenderichtlinie in Europa », ifo Schnelldienst, 4/2008

SCHRCEDER W., "Le modèle syndical allemand n'existe plus ", in BouRGEOIs I. (dir.), Le modèle social allemand en mutation, Cergy-Pontoise, 2005, et "Des relations sociales en plein bouleversement », Regards sur l'économie allemande, n`83/07

WEINKOPF C., Mindestbedingungen für die Zeitarbeitsbranche ?, IAT-Expertise, nov. 2006 WÖLFLE T., " Gewerkschaftliche Strategien in der Leiharbeit », WSI-Mitteilungen, 1/2008

ZUMFELDE M., "L'assouplissement du droit allemand des licenciements ", in BouRGEOIS I. (dir.), Le modèle social allemand en mutation, Cergy-Pontoise, 2005,

Zehnter Bericht der Bundesregierung über Erfahrungen bei der Anwendung des Arbeitnehmerüberlassungsgesetzes, Deutscher Bundestag, BT-Drucksache 15/6008, 2005

«Zeitarbeit : Barometer für den Arbeitsmarkt », iwd, 14/2008 